\title{
Handwriting Based Gender Classification Using COLD and Hinge Features
}

\author{
Abdeljalil Gattal $^{1(\bowtie)}$, Chawki Djeddi ${ }^{1,2}$, Ameur Bensefia $^{3}$, \\ and Abdellatif Ennaji ${ }^{2}$ \\ ${ }^{1}$ Department of Mathematics and Computer Science, Larbi Tebessi University, \\ Tebessa, Algeria \\ \{abdeljalil.gattal,c.djeddi\}@univ-tebessa.dz \\ ${ }^{2}$ LITIS Laboratory, University of Rouen, Rouen, France \\ abdel.ennaji@univ-rouen.fr \\ ${ }^{3}$ Higher Colleges of Technology, CIS Division, Abu Dhabi, UAE \\ abensefia@hct.ac.ae
}

\begin{abstract}
Gender Classification from handwriting is still considered to be challenging due to homogeneous vision comparing male and female handwritten documents. This paper presents a new method based on Cloud of Line Distribution (COLD) and Hinge feature for distinguishing the gender from handwriting. The SVM classifier combination decides the assigned class based on the maximum of the two decisions values resulting from COLD and Hinge feature. The proposed approach is evaluated on the standard QUWI dataset and following the framework protocol described in the ICFHR 2016 competition. Obtained results are promising regarding the classification rates announced in the literature.
\end{abstract}

Keywords: Handwriting $\cdot$ COLD feature $\cdot$ Hinge feature $\cdot$ Gender classification $\cdot$ SVM classifier

\section{Introduction}

The automated off-line handwriting analysis is one of the most researched problems in pattern recognition, due to its wide variety of applications, such as text recognition, writer identification [1], script identification [2], gender classification [3-8] and much more.

The automatic classification of gender-based on handwritten samples is the task that allows distinguishing between the writer's samples produced by males from those produced by females. Some psychological studies [9-11] confirmed that we could distinguish between the two genders writings thanks to some differences; where in general female handwritings tend to be a more uniform and regular, while male handwritings tend to be spikier and slanted. This task has attracted a lot of interest due to its application in forensic document examination, where it allows the examiners to focus on a particular category of writers. Thus, a handwriting based gender recognition system can be used in combination with a writer recognition system to process only part of the dataset, which may improve the processing rate in terms of performance and 
time. From handwriting variability point of view, the gender classification is in the middle, between handwriting recognition; where the variability needs to be blurred to emerge the common characteristic across all writers, and the writer identification, where the variability needs to be highlighted to arise differences between all writers. Therefore, in a gender recognition task, we need to emerge the handwriting variability, but not as much as in the writer identification, and not too low as in the handwriting recognition, since we are looking to find a common feature between a particular group of writers (males vs females), which makes this task delicate. Despite the fact, that the gender classification has received a lot of attention in the document analysis community [3-8] most of the research has been conducted by enhancing the feature extraction or/and classification process. In general, the gender classification problem makes a challenging task for a two-class problem. The study aims to focus on the enhancement of the feature extraction step to improve the classification rates on off-line handwritten documents.

In this paper, we present an original set of global features designed for gender recognition task: Cloud of Line Distribution (COLD) feature and Hinge features, coupled with two dedicated SVM classifiers for each set. We demonstrate through this study, how a maximum value of both SVM decisions; for COLD and Hinge features, can achieve high accuracy rates on handwriting-based gender recognition. Furthermore, the paper analyses how current state-of-the-art methods in gender classification perform on handwritten document dataset of ICFHR 2016 competition [12]. The best approach proposed in this competition was the "MCS-NUST", which uses Histogram of Local Binary Patterns (LBP), Histogram of Oriented Gradients (HoG) and Graylevel Co-occurrence Matrices (GLCM) to extract features from binarized images. These features trained using the SVMs classifier as well as SVM ensembles with bagging. Thus, we settled the ICFHR 2016 competition as a framework of comparison between our approach and those proposed in this event [12-14] where different evaluations mode have been performed (single and muti-scripts).

The paper is organized as follows: first, we present a literature review about some of the significant works conducted in gender recognition based on handwriting. Second, we detail our methodology where the features and the classifiers employed in our study are discussed. Section 4, details the experiments conducted along with a comparative analysis and discussion on the realized results. Finally, we conclude the paper with a discussion on future perspectives on the subject.

\section{Literature Review}

In this section, we present an exhaustive list of works that dealt with automatic gender classification based on handwriting. Since the task of gender classification is a recognition task, we focused on the elementary modules of such systems: features and classifier, in addition to the dataset used to evaluate the proposed system. In general, most of the proposed works articulate their methodologies either on local features and/or global features.

The authors in [6] proposed an approach that focuses on individual classification based on their age, nationality or gender. The formulation of this problem has been 
considered the same, whatever is the classification criterion. In other words, the same set of features and classification have been used in all cases. To this end, the authors proposed a set of features made of: direction, curvature, tortuosity, chain code and edge-based direction; combined with a Random Forest classifier (RF) and a kernel discriminant analysis (KDA). A rate of $74.05 \%$ in gender prediction was achieved when all the features where used with in QUWI dataset.

Another approach, in gender recognition, where local features were preferred to global ones, is presented in [5]. The Histogram Oriented Gradient (HOG) and the Local Binary Pattern (LBP) and grid features were used. A similar approach proposed in [15], where the same set of features was used, in addition to a feature resulting from a segmentation-based fractal texture analysis (SFTA) and features extracted from graylevel co-occurrence matrices (GLCM). The approach was evaluated on the QUWI dataset with a different combination of classifiers (SVM, ANN, KNN) and features. The best rate obtained by the authors is $85 \%$ when using all features with the three classifiers.

In [7] the gender classification has been tackled with the usage of global features; wavelet transform and symbol dynamic filtering (SDF). Firstly, the handwritten document is decomposed into sub-bands using the discrete wavelet transforms (DWT). These sub-bands are then extended into data-sequences; where a maximum entropy partitioning is applied. Finally, a features vector is built by concatenating all the SDF features obtained by the algorithm described in [16]. Two different datasets were used alternatively, for training and test, the QUWI and the MSHD dataset [17], combined with two different classifiers: ANN and SVM. The best gender recognition rate announced by the authors is $80 \%$. Liwicki et al. [4] also stated that gender classification could be successful with global features: Gaussian Mixture Models (GMM). In their methodology, offline and online features were used, since they processed the two types of handwritten documents. Even though the online gender classification is out of the scope of our paper, we consider that it is interesting to mention the impact of additional features in the performances rates. The system was evaluated with the IAM dataset, where 100 samples were used for training and validation and 50 samples for testing (equally distributed between males and females). Among the different combination of features used, the authors reached $67.75 \%$ as the best classification rate where both online and offline features were used and only $55.39 \%$ with the offline features.

Recently, gender classification from offline multi-script handwriting images using Oriented Basic Image Features (oBIFs) has been applied on handwritten document dataset of ICFHR 2016 competition [18]. The authors focused on the textural information in handwriting using combinations of different configurations of oBIFs histograms and oBIFs columns histograms. These features were used to train an SVM classifier and have been evaluated on the QUWI dataset, where $76 \%$ of good recognition rate was reached.

Previous works have shown that the texture features can improve the performance of gender classification system. We assume that the shape information and the curvature-based information provided by Hinge and COLDs features can characterize the writing style. 


\section{Proposed Methodology}

Most of the works conducted on gender classification are continuity of works undertaken in the writer's identification, where almost the same features were used. We consider that this assumption can be valid if we use global features rather than local features. Indeed, global features, even if they have discriminant power, they tend to emerge similarities between writings, which make them suitable for the gender recognition task. Therefore, in this section, we aim to design a new method based on Cloud of Line Distribution (COLD) and Hinge feature to capture female or male writing styles of handwritten documents (See Fig. 1).

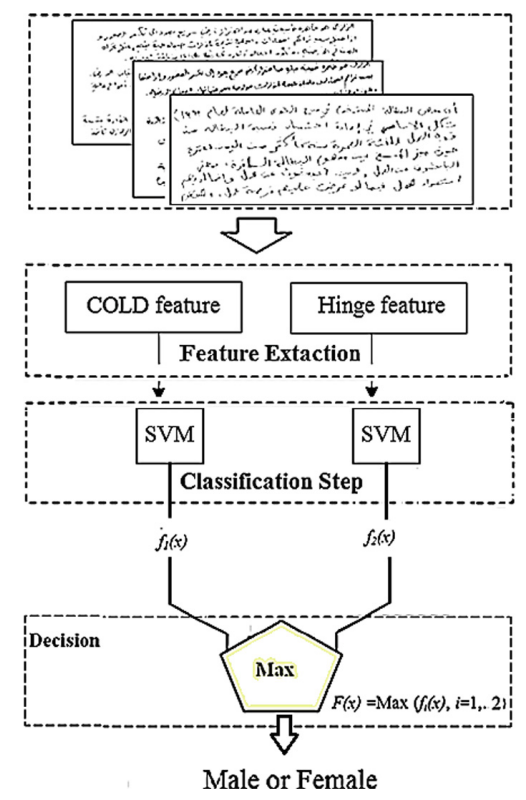

Fig. 1. Overview of the proposed system

\subsection{Features Extraction}

In our proposed methodology of gender classification, we have selected to use two sets of features: COLD feature and Hinge feature. This set of features has been successfully applied to the writer identification problem [19, 20]. In our study, we will take advantage of their strong discriminatory representation captured through the curvature information and contour information embedded in the handwritten document. The subsections below provide a detailed description of these features.

\section{COLD Feature}

The COLD feature [19] is inspired by the shape context descriptor, with respect to the extracted information into a log-polar histogram, which is more sensitive to regions of nearby the center than to those farther away [21]. The feature extracts unique shapes of 
handwritten text components by analyzing the relationship between dominant points; such as straight, angle-oriented features and curvature over contours of handwritten text components. Although, the COLD captures the writing style and the variations between different handwriting samples from the same gender by comparing the COLDs using the dominant points.

The log-polar space is based on three parameters: the distance between two consecutive rings in the log space $\mathrm{Dc}$, number of angular intervals $\mathrm{Np}$, and the number of distance intervals Nq. In our experimentations, we have empirically set these parameters as: $\mathrm{Dc}=5, \mathrm{~Np}=12$, and $\mathrm{Nq}=7$ [19]. Besides, the combination of COLDs with different $\mathrm{k}$ (level of connection between dominant points) achieves the best performance. Therefore, the ultimate COLD feature is generated by concatenating the COLDs with different $\mathrm{k}$ together.

\section{Hinge Feature}

The contour-based feature was designed to capture the curvature of the ink trace of the document images, which is considered to be very discriminatory between handwritings. The best contour-based features reported in the literature are the Hinge [22], QuillHinge [23], and Delta-n Hinge features [20].

The Hinge feature [22] is the probability distribution of orientations of two legs of the obtained "hinge" based on edges or contours together attached at a current pixel. There are two parameters in the Hinge feature: the number of angle bins $p$ and the leg length $r$. In our case, we set $p=40$, as suggested in [23]. Therefore, it has been extended to the Delta-n Hinge [23] to achieve the rotation-invariant property. There are four parameters in the proposed method, the number of angle bins $p$, leg length $r$, Manhattan distance $\Delta \mathrm{l}$, and the number of derivative $\mathrm{n}$. In our experimentations we set $\mathrm{p}=40$, the Manhattan distance $\Delta \mathrm{l}=7$, and $\mathrm{n}=2$.

The two sets of features COLD and Hinge are combined only at the decision level. In other words, we proceed with the gender classification by submitting each set of features to a separate classifier, as discussed in the following section.

\subsection{Decision Strategy}

In an attempt to enhance the reliability of the accuracy rates of the proposed system, a decision module is designed to produce the final decision. This module is proposed with two different SVM classifiers [24], where each classifier is trained by one set of features: COLD or Hinge. The training of each SVM requires the selection of two parameters, which are the regularization parameter $\mathrm{C}$ and the Radial Basis Function (RBF) kernel parameter $(\sigma)$ is selected in the range $\sigma=[1: 50]$, with the soft margin parameter $\mathrm{C}$ fixed to 10 , which allows improvement in the accuracy rate. The choice of hyperparameters values selected by experimentation to achieve better performance while overcoming over fitting to avoid identity or isometric identity kernel matrix. However, there is no robust rule available to guide how to choose the hyper parameters values appropriately. The final decision of our module is taken by selecting the maximum value of the decision function produced by the two SVM classifiers. Experimentally, the maximum (Max) decision resulting from COLD and Hinge features achieved the best performance compared to other standard statistical reasoning 
measures such as average (Avr) and minimum (Min). In the next section, we present the experimental settings and the corresponding results.

\section{Experimentations and Results}

We carried out a series of experiments to evaluate the effectiveness of the proposed system for gender classification from handwriting on competition datasets.

A subset of QUWI dataset, used in ICFHR 2016 competition [12, 25], comprised 1000 handwritten documents samples were selected: 500 samples were provided as a training set, 250 as the validation set and 250 as a test set. The most interesting aspect of this competition is the usage of a dataset with handwritten documents of the same writer in multiple scripts, and also the opportunity to study the performance of scriptdependency (Sub-task 2A and Sub-task 2B) and script-independency (Sub-task 2C and Sub-task 2D) in a multi-script experimental setup. Our experiments aim to study the effect of different $k$ combination of COLDs, and various leg length $r$ from Hinge and Delta-n Hinge features, while the number of angle bins $\mathrm{p}=40$. The realized classification rates are illustrated in Fig. 2 and Fig. 3.

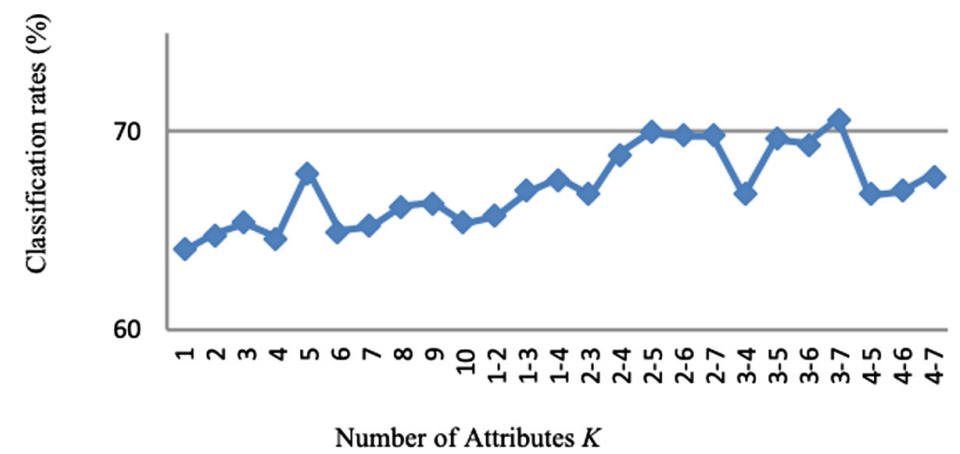

Fig. 2. Classification rates on both scripts QUWI dataset used in ICFHR 2016 competition using COLD feature and its combination.

It can be seen that the combination of COLDs features while $k=\{3,4,5,6,7\}$ outperforms the other COLDs features. Therefore, the Hinge feature with $r=25$ while $\mathrm{p}=40$ does better than others Hinge and Delta-n Hinge features configurations. The combination of COLDs features is extracted from the complete handwriting image as well as they are obtained by applying the Uniform Grid Sampling (UGS) [26] to the handwriting; this allows extracting features from different regions of the image separately.

According to the previous results, we evaluated the proposed method using the decision strategy with optimal features on both scripts (all samples in Arabic and English). Table 1 reports the different features and the proposed method. 


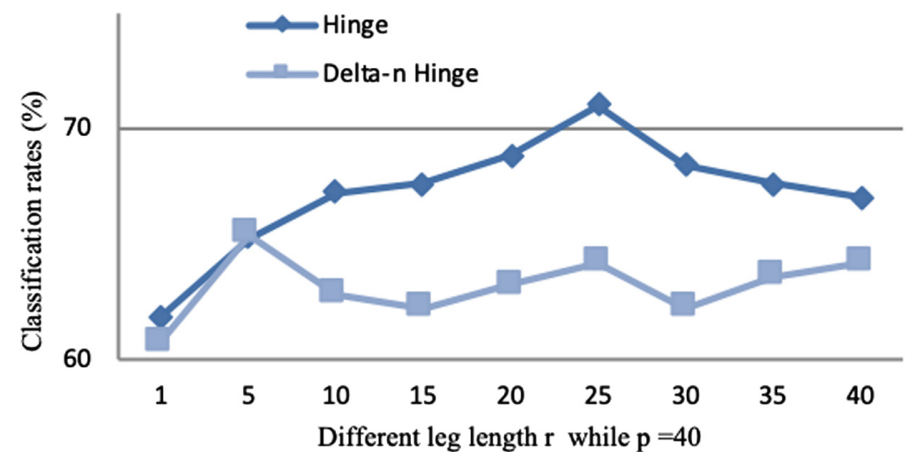

Fig. 3. Classification rates on both scripts QUWI dataset used in ICFHR 2016 competition using hinge feature and Delta-n Hinge feature.

Table 1. Gender classification rates with different features the proposed method

\begin{tabular}{l|l|l|l|l|l}
\hline \multicolumn{2}{l|}{ Features description } & Feature parameters & UGS & Dim & Classification rates (\%) \\
\hline F1 & Hinge & $\mathrm{r}=25, \mathrm{p}=40$ & No & 780 & 71.00 \\
\hline F2 & Deltahinge & $\mathrm{r}=5, \mathrm{p}=40$ & No & 780 & 65.40 \\
\hline F3 & COLD feature & $\mathrm{k}=5$ & No & 84 & 68.00 \\
\hline F4 & COLDs features Combination & $\mathrm{k}=3,4,5,6,7$ & No & 420 & 70.60 \\
\cline { 3 - 6 } F5 & $\mathrm{k}=3,4,5,6,7$ & $1 \mathrm{x} 2$ & 840 & 71.00 \\
\hline \multicolumn{2}{l}{ Proposed method } & & & & 73.60 \\
\hline
\end{tabular}

It can be seen that the maximum value on the decision functions produced by COLDs features extracted from the $1 \times 2$ regions of the image while $k=\{3,4,5,6,7\}$ and the Hinge feature with $r=25$ while $p=40$ outperforms all other features reporting classification rates of $73.60 \%$ on both scripts. Furthermore, we use our proposed methodology according to the competition protocol in script-dependent and scriptindependent evaluations. According to sub-task $2 \mathrm{~A}$ and sub-task $2 \mathrm{~B}$ for Scriptdependent evaluation described in the ICFHR2016 competition, the protocol is performed using 500 script samples in the training set and 250 same script samples (English (En) or Arabic (Ar)) in the test set. The results of our proposed approach with different ones in Script-dependent evaluation are summarized in Table 2.

It can be seen from Table 2 that our approach realizes an average rate of $74.20 \%$, which is comparable to performances of the top 2 systems, according to the competition protocol. However, it should be noted that the curvature and contour information used in our method can compete for the textural information [18]. These results validate the effectiveness of the proposed method in Script-dependent evaluation mode.

According to sub-task 2C and sub-task 2D in Script-independent evaluation, the experiments is performed using 500 script samples in the training set and 250 other script samples in the test set. Table 3 shows that the classification rates of our proposed approach in script-independent evaluation compared with different approaches. 
Table 2. Classification rates of script-dependent evaluations.

\begin{tabular}{|c|c|c|c|c|c|}
\hline \multirow[t]{2}{*}{ Method } & \multicolumn{2}{|l|}{ Script } & \multirow[t]{2}{*}{ Classifier } & \multicolumn{2}{|c|}{ Classification rates $(\%)$} \\
\hline & Train & Test & & Script dependent & Average \\
\hline \multirow[t]{2}{*}{ Proposed method } & $\mathrm{Ar}$ & $\mathrm{Ar}$ & \multirow[t]{2}{*}{ SVM } & 74.80 & \multirow[t]{2}{*}{74.20} \\
\hline & En & En & & 73.60 & \\
\hline \multirow[t]{2}{*}{ Gattal et al. [18] } & $\mathrm{Ar}$ & $\mathrm{Ar}$ & \multirow[t]{2}{*}{ SVM } & 74.80 & \multirow[t]{2}{*}{75.00} \\
\hline & En & En & & 75.20 & \\
\hline \multirow[t]{2}{*}{ MCS-NUST1 method [12] } & $\mathrm{Ar}$ & $\mathrm{Ar}$ & \multirow[t]{2}{*}{ SVM } & 61.60 & \multirow[t]{2}{*}{58.80} \\
\hline & En & En & & 56.00 & \\
\hline \multirow[t]{2}{*}{ MCS-NUST2 method [12] } & $\mathrm{Ar}$ & $\mathrm{Ar}$ & \multirow[t]{2}{*}{ SVM with bagging } & 60.80 & \multirow[t]{2}{*}{59.20} \\
\hline & En & En & & 57.60 & \\
\hline \multirow[t]{2}{*}{ Nuremberg1 method [12] } & $\mathrm{Ar}$ & $\mathrm{Ar}$ & \multirow[t]{2}{*}{ SVM } & 58.00 & \multirow[t]{2}{*}{56.00} \\
\hline & En & En & & 54.00 & \\
\hline \multirow[t]{2}{*}{ Nuremberg2 method [12] } & $\mathrm{Ar}$ & $\mathrm{Ar}$ & \multirow[t]{2}{*}{ SVM } & 46.40 & \multirow[t]{2}{*}{60.20} \\
\hline & En & En & & 74.00 & \\
\hline
\end{tabular}

Table 3. Classification rates of script-independent evaluations.

\begin{tabular}{|c|c|c|c|c|c|}
\hline \multirow[t]{2}{*}{ Method } & \multicolumn{2}{|l|}{ Script } & \multirow[t]{2}{*}{ Classifier } & \multicolumn{2}{|c|}{ Classification rates $(\%)$} \\
\hline & Train & Test & & Script independent & Average \\
\hline \multirow[t]{2}{*}{ Proposed method } & $\mathrm{Ar}$ & En & \multirow[t]{2}{*}{ SVM } & 64.00 & \multirow[t]{2}{*}{64.40} \\
\hline & En & $\mathrm{Ar}$ & & 64.80 & \\
\hline \multirow[t]{2}{*}{ Gattal et al. [18] } & $\mathrm{Ar}$ & En & \multirow[t]{2}{*}{ SVM } & 66.00 & \multirow[t]{2}{*}{68.00} \\
\hline & En & $\mathrm{Ar}$ & & 70.00 & \\
\hline \multirow[t]{2}{*}{ MCS-NUST1 method [12] } & $\mathrm{Ar}$ & En & \multirow[t]{2}{*}{ SVM } & 57.60 & \multirow[t]{2}{*}{58.60} \\
\hline & En & $\mathrm{Ar}$ & & 59.60 & \\
\hline \multirow[t]{2}{*}{ MCS-NUST2 method [12] } & $\mathrm{Ar}$ & En & \multirow[t]{2}{*}{ SVM with bagging } & 58.40 & \multirow[t]{2}{*}{58.80} \\
\hline & En & Ar & & 59.20 & \\
\hline \multirow[t]{2}{*}{ Nuremberg1 method [12] } & $\mathrm{Ar}$ & En & \multirow[t]{2}{*}{ SVM } & 56.00 & \multirow[t]{2}{*}{58.60} \\
\hline & En & $\mathrm{Ar}$ & & 61.20 & \\
\hline \multirow[t]{2}{*}{ Nuremberg2 method [12] } & $\mathrm{Ar}$ & En & \multirow[t]{2}{*}{ SVM } & 72.40 & \multirow[t]{2}{*}{58.80} \\
\hline & En & $\mathrm{Ar}$ & & 45.20 & \\
\hline
\end{tabular}

We notice that the proposed methodology achieves higher average rate in scriptindependent evaluations than other methods in the literature, except Gattal et al. [18] method. The average classification rate is $64.40 \%$. We clearly note that the proposed method is in the top 2 methods, according to the competition protocol in scriptdependent and script-independent evaluations. Besides, in some cases, our proposed method can provide unsuccessful classification results. As example, male handwritten documents found to have a homogeneous vision comparing to female handwritten documents and vice versa. 


\section{Conclusions and Future Works}

An effective approach for characterizing gender based on their handwriting is presented, which that exploits COLD and Hinge as features. Different combinations of the selected features are investigated using an SVM classifier. The system is evaluated using the same experimental protocol described in the ICFHR2016 Competition on Multi-script Writer Demographics Classification. The results showed that our approach outperforms the existing methods reported in the mentioned competition, except the new method proposed by Gattal et al. [18].

In our further study on this problem, we intend to investigate other kinds of features to characterize the gender from handwriting by exploring feature selection techniques to identify the most appropriate descriptors for this problem. Finally, we also plan to determine different demographic traits, such as age and handedness from handwriting.

\section{References}

1. Srihari, S., Cha, S.-H., Arora, H., Lee, S.: Individuality of handwriting. J. Forensic Sci. 47, 856-872 (2002)

2. Singh, P.K., Sarkar, R., Nasipuri, M.: Offline script identification from multilingual Indicscript documents: a state-of-the-art. Comput. Sci. Rev. 15-16, 1-28 (2015)

3. Siddiqi, I., Djeddi, C., Raza, A., Souici-meslati, L.: Automatic analysis of handwriting for gender classification. Pattern Anal. Appl. 18(4), 887-899 (2015)

4. Liwicki, M., Schlapbach, A., Bunke, H.: Automatic gender detection using on-line and offline information. Pattern Anal. Appl. 14(1), 87-92 (2011)

5. Bouadjenek N., Nemmour H., Chibani Y.: Local descriptors to improve off-line handwriting-based gender prediction. In: 2014 6th International Conference of Soft Computing and Pattern Recognition (SoCPaR), pp. 43-47 (2014)

6. Al Maadeed, S., Hassaine, A.: Automatic prediction of age, gender, and nationality in offline handwriting. EURASIP J. Image Video Process. 2014(1), 1-10 (2014). https://doi.org/10. 1186/1687-5281-2014-10

7. Akbari, Y., Nouri, K., Sadri, J., Djeddi, C., Siddiqi, I.: Wavelet-based gender detection on off-line handwritten documents using probabilistic finite state automata. Image Vis. Comput. 59, 17-30 (2017)

8. Mirza, A., Moetesum, M., Siddiqi, I., Djeddi, C.: Gender classification from offline handwriting images using textural features. In: 2016 15th International Conference on Frontiers in Handwriting Recognition (ICFHR), pp. 395-398 (2016)

9. Goodenough, F.L.: Sex differences in judging the sex of handwriting. J. Soc. Psychol. 22(1), 61-68 (1945)

10. Hartley, J.: Sex differences in handwriting: a comment on spear. Br. Educ. Res. J. 17(2), 141-145 (1991)

11. Burr, V.: Judging gender from samples of adult handwriting: accuracy and use of cues. J. Soc. Psychol. 142(6), 691-700 (2002)

12. Djeddi, C., Al-Maadeed, S., Gattal, A., Siddiqi, I., Ennaji, A., Abed, H.E.: ICFHR2016 competition on multi-script writer demographics classification using 'QUWI' database. In: 2016 15th International Conference on Frontiers in Handwriting Recognition (ICFHR), pp. 602-606 (2016) 
13. Hassaïne, A., Al Maadeed, S., Aljaam, J., Jaoua, A.: ICDAR 2013 competition on gender prediction from handwriting. In: 2013 12th International Conference on Document Analysis and Recognition, pp. 1417-1421 (2013)

14. Djeddi C., Al-Maadeed S., Gattal A., Siddiqi I., Souici-Meslati L., El Abed H.: ICDAR2015 competition on multi-script writer identification and gender classification using 'QUWI' database. In: 2015 13th International Conference on Document Analysis and Recognition (ICDAR), pp. 1191-1195 (2015)

15. Ahmed, M., Rasool, A.G., Afzal, H., Siddiqi, I.: Improving handwriting based gender classification using ensemble classifiers. Expert Syst. Appl. 85, 158-168 (2017)

16. Bahrampour, S., Ray, A., Sarkar, S., Damarla, T., Nasrabadi, N.M.: Performance comparison of feature extraction algorithms for target detection and classification. Pattern Recognit. Lett. 34(16), 2126-2134 (2013)

17. Djeddi, C., Gattal, A., Souici-Meslati, L., Siddiqi, I., Chibani, Y., El Abed, H.: LAMISMSHD: a multi-script offline handwriting database. In: 2014 14th International Conference on Frontiers in Handwriting Recognition, pp. 93-97 (2014)

18. Gattal, A., Djeddi, C., Siddiqi, I., Chibani, Y.: Gender classification from offline multi-script handwriting images using oriented Basic Image Features (oBIFs). Expert Syst. Appl. 99, 155-167 (2018)

19. He, S., Schomaker, L.: Writer identification using curvature-free features. Pattern Recognit. 63, 451-464 (2017)

20. He S., Schomaker L.: Delta-n Hinge: rotation-invariant features for writer identification. In: 2014 22nd International Conference on Pattern Recognition, pp. 2023-2028 (2014)

21. Belongie, S., Malik, J., Puzicha, J.: Shape matching and object recognition using shape contexts. IEEE Trans. Pattern Anal. Mach. Intell. 24(4), 509-522 (2002)

22. Bulacu, M., Schomaker, L.: Text-independent writer identification and verification using textural and allographic features. IEEE Trans. Pattern Anal. Mach. Intell. 29(4), 701-717 (2007)

23. Brink, A.A., Smit, J., Bulacu, M.L., Schomaker, L.R.B.: Writer identification using directional ink-trace width measurements. Pattern Recogn. 45(1), 162-171 (2012)

24. Apnik, V.N.: The Nature of Statistical Learning Theory. Springer, Heidelberg (1995). https://doi.org/10.1007/978-1-4757-2440-0

25. Maadeed, S.A., Ayouby, W., Hassaïne, A., Aljaam, J.M.: QUWI: an Arabic and English handwriting dataset for offline writer identification. In: 2012 International Conference on Frontiers in Handwriting Recognition, pp. 746-751 (2012)

26. Gattal, A., Chibani, Y., Djeddi, C., Siddiqi, I.: Improving isolated digit recognition using a combination of multiple features. In: 2014 14th International Conference on Frontiers in Handwriting Recognition, pp. 446-451 (2014) 